\title{
Les évangéliques cartographient le monde
}

Le spiritual mapping

Nadège Mézié

\section{(2) OpenEdition}

Journals

Édition électronique

URL : http://journals.openedition.org/assr/13973

DOI : $10.4000 /$ assr. 13973

ISSN : 1777-5825

Éditeur

Éditions de l'EHESS

\section{Édition imprimée}

Date de publication : 1 juin 2008

Pagination : 63-85

ISBN : 978-2-7132-2190-3

ISSN : 0335-5985

Référence électronique

Nadège Mézié, "Les évangéliques cartographient le monde ", Archives de sciences sociales des religions [En ligne], 142 | avril-juin 2008, mis en ligne le 20 octobre 2011, consulté le 19 avril 2019. URL : http://journals.openedition.org/assr/13973 ; DOI : 10.4000/assr.13973 


\section{Nadège Mézié}

\section{Les évangéliques cartographient le monde Le spiritual mapping}

Prendre pour objet l'entreprise missionnaire chrétienne conduit d'une manière ou d'une autre à un ensemble de notions qui lui sont intimement associées et qui ont trait à la définition que l'on accorde au terme de mission. David Bosch et Claude Prudhomme insistent dans leurs ouvrages respectifs sur le fait que la mission a pris, à travers le temps, des significations différentes et que le mot peut recouvrir plusieurs connotations. David Bosch recense ainsi huit significations récemment instituées parmi lesquelles « envoi de missionnaires dans un territoire bien précis ; (...) les activités entreprises par ces missionnaires ; (...) aire géographique où ils ouvraient ; (...) monde non chrétien, le "champ de mission" (...) » (Bosch, 1995 : 11). Plusieurs de ces significations renvoient donc à la dimension extérieure, exotique de la mission (pour la différencier de la mission intérieure) et convoquent à sa suite une pléiade de termes comme ceux de voyage, de colonisation, d'ailleurs, de territoire ou encore d'espace. L'action apostolique dirigée vers les non-chrétiens suppose la visualisation d'un espace étranger, la mobilité, le déplacement, l'insertion et l'inscription dans un territoire. Dès lors, il n'est pas étonnant que, très tôt, des chrétiens fervents prosélytes se soient consacrés à l'élaboration de mappemondes, de portulans, de cartes. La cartographie élaborée à travers les siècles par ces chrétiens nous offre ainsi un angle d'approche original et une lecture heuristique du cadre complexe qu'est l'entreprise missionnaire chrétienne où l'imaginaire et le politique viennent se greffer au religieux et s'invitent sous la forme de schèmes activateurs d'action.

Nous proposons au lecteur une brève histoire de la cartographie chrétienne et nous nous intéresserons en dernier lieu à une cartographie évangélique contemporaine, le spiritual mapping, qui recoupe en fait deux champs de connaissances et d'actions qu'il nous faut clairement différencier tandis qu'une seule de ces versions, à travers le Joshua Project, retiendra notre attention. En intégrant la cartographie spirituelle élaborée principalement par des évangéliques américains à la longue durée, nous voulons tenter de comprendre les mécanismes à l'œuvre dans l'élaboration des cartes " chrétiennes » afin de faire émerger les topoi disparus, récurrents ou inédits et de saisir les divers usages qu'il a été fait de ces cartes. 
64 - ARCHIVES DE SCIENCES SOCIALES DES RELIGIONS

C'est pourquoi, il nous faut envisager la carte comme un objet d'étude à part entière car, loin d'être muettes, les cartes nous parlent de savoirs, de conceptions symboliques de l'espace et de l'autre, d'intentions, de pouvoirs, d'angoisses et d'espoirs.

\section{Jalons pour une histoire de la " cartographie chrétienne "}

Avant de nous tourner vers les siècles passés, il nous faut donner une définition de la "cartographie chrétienne " afin de délimiter notre objet. Par cette expression nous entendons un dispositif de savoirs (géographiques, religieux...) concrétisé dans une carte (ou une mappemonde ou un planisphère) commanditée et/ou réalisée par des chrétiens selon une ou des finalité(s) précise(s) qui varient selon le contexte spatio-temporel (glorification du nom de Dieu et édification du chrétien dans les mappemondes médiévales ou détermination de l'action missionnaire dans les cartes contemporaines des organisations évangéliques). Dans tous les cas, la représentation d'un espace ne peut être considérée comme une donnée naturelle, au sens où elle serait représentation en tous points fidèle et mimétique de la réalité mais doit, au contraire, être appréhendée comme poiesis, c'est-à-dire un objet de création et comme toute création, elle cristallise des intentionnalités, plus ou moins conscientes, plus ou moins motivées, elle habite des valeurs ${ }^{1}$, elle est l'expression d'un état donné de savoirs, de schèmes symboliques.

Le jalonnement que nous présentons au lecteur se veut aiguillage vers des périodes clés de la "cartographie chrétienne ». Plus qu'une histoire détaillée, nous voulons proposer des pistes grossièrement défrichées mais qui laissent entrevoir la richesse de notre sujet. Nous voulons insister sur le fait que le spiritual mapping s'inscrit dans une tradition géographique et cartographique religieuse bien ancrée qui se révèle dès les premiers temps du christianisme. L'intérêt des chrétiens pour ces disciplines est d'autant plus motivé et vif que le christianisme dans son essence même est une religion missionnaire, tournée vers les terres de l'ailleurs.

\section{L'évangile aux confins de la terre}

Le règne de Dieu au temps de Moïse s'incarnait dans l'alliance avec les tribus juives, centrée sur Jérusalem. A contrario, Jésus, en énonçant le Grand Commandement «Allez, faites de toutes les nations des disciples » (Mt 28,19), déterritorialise l'alliance : tout homme sur la terre (œkoumène), juif ou païen est potentiellement élu dans l'universel peuple de Dieu. L'Église du ressuscité - Jésus est mort et ressuscité pour tous -, a vocation à l'universel (katholikos) et n'est

1. Comme le rappelle Wittgenstein, le monde en soi est dénué de valeurs, c'est l'homme qui vient lui en apposer. 
plus enraciné dans la Synagogue ${ }^{2}$. L'homme, renouvelé dans le Saint-Esprit (instruit, baptisé et obéissant aux prescriptions morales), répond avec Ésaie " Me voici, envoie-moi » (Es 6-8). Aux nations, il témoigne de la vérité, de l'amour et de l'unité de Dieu (Jean 17) et s'offre à l'œuvre de propagation de la Bonne nouvelle avec les mots du premier missionnaire « Je me dois» (Rom 1,14).

Le christianisme est donc une religion missionnaire et c'est pourquoi «le christianisme apporta une immense révolution, (...) il récuse les dieux autres que le sien non seulement pour lui, mais pour tout le monde. Son dieu ne se borne pas à être le dieu de ses croyants, il est celui de l'Être en général. (...) Le dieu chrétien est le premier à étendre sa sphère sur ceux qui croient en lui comme sur ceux qui n'y croient pas» (Simmel, $1998: 94$ ).

\section{Les mappemondes ou la création divine}

Les premières «cartes chrétiennes " n'ont pas pour finalité de donner au propagateur de la Parole de Dieu les éléments de localisation et de repérage des lieux mais se présentent comme les instruments de l'édification du chrétien et de la glorification du Dieu créateur. Ces mappemondes sont soumises à la théologie - Nathalie Bouloux parle ainsi de mappemondes "théologiques » -, et participent de l'enseignement chrétien en montrant, par exemple, l'histoire humaine depuis la genèse, en évoquant les préceptes moraux.

Les cartes dessinées pour les mappemondes s'élaborent le plus souvent selon des significations allégoriques. Ainsi, Constantin d'Antioche, dit Cosma Indicopleuste ( $\mathrm{VI}^{\mathrm{c}}$ siècle), sous l'influence de la théorie nestorienne et contre la théorie aristotélicienne de la sphéricité du monde, défend le modèle d'un cosmos à l'imitation du tabernacle dicté par Dieu à Moïse $(\mathrm{Ex} 26)^{3}$. Un programme cosmologique (surimpression des nouvelles idées chrétiennes sur les éléments byzantins classiques) que l'on retrouve à l'œuvre dans les édifices ecclésiastiques byzantins. Façonné de théories chrétiennes et mystiques, Cosma ne tira que peu partie de ses expériences maritimes. "C'est la structure du mythe - non l'observation proprement dite - qui va organiser les données et contribuer à les rendre plus crédibles » (Kilani, $2000: 66$ ). Quelques siècles plus tard, Hugues de Saint-Victor décrit le monde sous la forme de l'arche de Noé (1128-1129) : il s'agit pour lui de «poser un »regard spirituel « sur le monde » (Lecoq, 1989: 22). L'image du monde est alors le reflet d'une conception du monde : « De nombreuses formes visibles représentant des concepts spirituels chrétiens sont évidentes dans les Mappamundi. Quelque fois c'est la carte entière qui est présentée comme le symbole des vérités chrétiennes »(Woodward 1987 : 334 ; la traduction est la nôtre).

2. Pour une analyse complète de l'impératif missionnaire dans l'évangile de Matthieu voir le chapitre "La mission dans l'évangile de Matthieu: une façon de faire des disciples" (Bosch, 1995).

3. Sur Cosma Indicopleuste voir de O.A.W. Dilke "Cartography in the Byzantine Empire " (1987 : 261-263). 


\section{Une cartographie pour une croisade tardive}

De nouveaux usages apparaissent au cours du bas Moyen Âge. Ainsi, le cartographe Petrus Vesconte élabore, vers 1320, une mappemonde pour Marino Sanudo Torsello, auteur du traité de croisade Liber secretorum fidelium crucis dont le but premier était de persuader le pape et les gouvernants d'organiser une nouvelle croisade. Cet ouvrage est à la fois un itinerarium peregrinationis, une pratica della mercatura, un portulan, un traité d'histoire-géographie et d'économie (financement des croisades) et une epitoma rei militaris (offrir les renseignements aux croisés pour mener à bien la guerre maritime). La mappemonde de Vesconte venait s'insérer dans une collection de plans, de cartes, de portulans que le vénitien Sanudo Torsello avait rassemblée afin d' " appuyer la stratégie de croisade et d'en assurer la faisabilité »(Bouloux, 2006:316). Une autre carte de Vesconte figure dans l'ouvrage, il s'agit d'une carte de la Palestine - qui fut un modèle pour la cartographie moderne - où figurent des références à l'Ancien Testament : on y trouve ainsi les douze tribus, une description de la tombe de Job tandis que les forts des croisés sont recensés le long des côtes. Si ces cartes opèrent un déplacement dans leur finalité et peuvent être considérées comme les précurseurs d'une cartographie à l'usage des missionnaires, elles ne conservent pas moins une propension à l'enseignement et à la moralisation de l'espace géographique $^{4}$.

\section{Les jésuites et l'enseignement géographique}

Alessandro Scafi voit un changement notable vers 1500 dans l'élaboration et la thématique des cartes. Il constate le passage d'un paradigme à l'autre, "d'une cartographie qui cherche à pénétrer le mystère de tout l'univers au-delà des frontières humaines à une cartographie qui est strictement limitée dans le cadre de la pensée analytique et de la géométrie euclidienne et d'une cosmographie à une géographie » (Scafi, 1999: 70). Ce passage d'un savoir piloté par la théologie, à une science géographique qui s'émancipe peu à peu de ses références religieuses entraîne l'affirmation d'une "cartographie chrétienne » entièrement dévouée à la cause des missionnaires. Les chrétiens, réalisateurs de cartes ou commanditaires, vont contribuer à l'élaboration d'une cartographie toujours plus précise et étendue tout en conservant un cadre religieux, un contexte qui n'a plus ou presque d'incidence sur le texte (la carte). C'est l'usage qui fait la carte « chrétienne».

4. On trouve une entreprise de moralisation à grande échelle dans les Grands Voyages, édition en treize volumes avec gravures des récits de voyage et de conquête de l'Amérique gravée et éditée par le huguenot Théodore de Bry (1528-1598). L'entreprise doit être lue au regard des guerres de religions, de Bry accentuant les atrocités des espagnols catholiques et valorisant le Bon Sauvage (Bouyer et Duviols, 1992). 
Les jésuites ont très vite compris l'intérêt de s'emparer de ce puissant instrument ainsi que nous le rappelle François de Dainville dans son livre passionnant sur la géographie des humanistes (Dainville, 1969). Il écrit "C'est vers les routes du Proche-Orient et l'Empire Turc que se tendent les regards d'Ignace de Loyola et de ses compagnons. Le Turc demeure l'objet des préoccupations du Pape et de la Chrétienté. (...) Plus des deux tiers [des ouvrages "géographiques" publiés en France à cette époque] ont pour sujet la Terre Sainte et l'empire des Turcs » (ibid.: 15). Certains jésuites se font cartographes, mais plus généralement l'appropriation de la cartographie va essentiellement s'opérer par l'enseignement de la géographie. Ils ont pour prédilection la géographie descriptive ou humaine, qui était d'un grand secours pour les moralistes, car elle s'attachait « de préférence à l'homme et aux manifestations humaines " (ibid.: 54). La géographie humaine, outre sa valeur moralisatrice, a pour tâche de donner des informations utiles aux jésuites en mission, elle doit leur fournir des renseignements sur les populations à évangéliser. Il faut rappeler avec D. Bosch que, jusqu'au XVI e siècle, le terme de mission ne se référait qu'à l'envoi du Fils par Dieu le père et « du Saint-Esprit par le Père et le Fils» (Bosch, 1995 : 11) ; ce sont les jésuites qui ont utilisé pour la première fois le mot de mission "pour exprimer l'extension de la foi chrétienne à des gens (...) qui n'étaient pas membres de l'Église catholique " (ibid. : 11). Les jésuites, à la suite de leur maître Ignace de Loyola, se sont faits apôtres des infidèles; en 1540, la bulle Regimini confirme l'Institut des jésuites dont la fin spécifique est l'activité missionnaire. Alors que dans les collèges $\mathrm{d}$ 'Europe se préparaient les missions, la géographie trouve toute sa valeur, "les exigences de la formation missionnaire durent peser en faveur des études de géographie mathématique " (ibid.: 106). Dainville rappelle que les murs des établissements étaient ornés de cartes et cite un passage très significatif d'un dialogue de 1556 qui nous dépeint des disciples d'Ignace réunis dans la maison de campagne du Collège romain assis "sur un banc (...) dans un petit pavillon (...) orné de quelques tableaux dévotieux et de diverses cartes de géographie, même des Indes et de Ternate, dont nous avions un peu auparavant reçu nouvelle certaine de la conversion des Îles du Japon (...) » (ibid. : 105). Dainville en conclut que les liens ont été très étroits dès le début entre la missiologie (l'art de faire des disciples) et la géographie et que les missionnaires ont été de ceux qui ont permis la rectification et l'élaboration d'une cartographie scientifique. Mais, il ne faudrait pas se méprendre : cette géographie descriptive, comme nous le rappelions plus haut, s'insère (contexte) dans une géographie sacrée (le récit biblique) comme le veut Antonio Possevino (1533-1611), secrétaire général des jésuites, lequel conseille au "professeur de géographie " de débuter "par des notions générales sur le globe : le Paradis terrestre après le déluge, le partage de la terre entre les fils de Noé » pour en venir «à la Palestine, à la Phénicie et aux frontières de la Terre Promise. On apprendra à connaître les lieux où se déroulent les événements rapportés par l'Écriture (...) » (ibid. : 57). 
68 - ARCHIVES DE SCIENCES SOCIALES DES RELIGIONS

\section{Les missions protestantes}

Ce n'est vraiment qu'à partir du XVII ${ }^{\mathrm{e}}$ siècle que les protestants, libérés du poids des conflits et des affrontements ${ }^{5}$, s'engagent dans le travail missionnaire ${ }^{6}$. Cette arrivée tardive sur le terrain missionnaire explique l'absence d'une " cartographie chrétienne " spécifiquement protestante. La première école missionnaire, le séminaire de Walaüs, est fondée en 1622 à Leyde (Pays-Bas) et forme les futurs missionnaires qui se destinent aux Indes néerlandaises. À leur tour, les piétistes font de l'Université de Halle (Allemagne), un centre de formation des missionnaires qu'ils envoient au Groenland, aux Indes... Pour autant, il faut attendre la fin $\mathrm{du} \mathrm{XVIII}^{\mathrm{c}}$ siècle et le début du $\mathrm{XIX}^{\mathrm{c}}$ siècle pour que s'opèrent un tournant décisif et une expansion mondiale favorisés à la fois par la seconde vague de colonisation, par les réveils religieux et par l'apparition du méthodisme. Les dénominations mainlines suivent leurs nations-mères à travers les mers. L'anglicanisme s'établit à la faveur des conquêtes britanniques en Australie, au Canada et en Nouvelle-Zélande. Mais ce sont les nouveaux courants religieux qui vont se montrer les plus ardents prosélytes. Ces courants qui apparaissent dès le $\mathrm{XVII}^{\mathrm{e}}$ siècle, jusqu'au pentecôtisme qui prend naissance au tout début du $\mathrm{XX}^{\mathrm{e}}$ siècle, et que l'on regroupe communément sous le terme d'évangéliques (vaste nébuleuse aux contours flous dans laquelle on peut inclure la composante piétiste, la composante orthodoxe - à tendance fondamentaliste -, et la composante charismatiquepentecôtiste), placent le prosélytisme au cœur de leur agir religieux. Pour David Bebbington, l'activisme forme, avec la conversion personnelle, la centralité de la Bible et l'insistance sur le Christ, le " quadrilatère des priorités qui sont la base de l'évangélisme »(Bebbington, 1989: 3). John Wesley (1703-1791), figure majeure du réveil méthodiste, se fait ainsi l'écho de cette volonté ardente de proclamer l'Évangile en s'exclamant "Le monde est ma paroisse ». Le baptisme n'est pas en reste et, sous l'influence d'Andrew Fuller (1754-1815) et de William Carey (1731-1834), s'implante sur les quatre continents. À la même époque, de nombreuses sociétés missionnaires sont créées et renforcent ce mouvement d'expansion ; signalons, entre autres, la Société néerlandaise des missions (1795), le Conseil américain des missions (1810), la Société des missions évangéliques de Paris (1822). «De 1792 à 1900, plus de trois cents sociétés missionnaires protestantes sont créées en Europe et en Amérique »(Baubérot, 2005 : 300).

5. D. Bosch développe dans les pages 319-336 les raisons intrinsèques et extrinsèques de l'absence d'ambition missionnaire chez les premiers réformateurs et conclut sur cette première période «Sur le plan de la théologie, [l’Église de la Réforme] était plus scolastique qu'apostolique » (Bosch $1995:$ 332).

6. Pour cet aperçu historique, je m'appuie sur l'article "Missions" par Jean Baubérot et Jean-Paul Willaime dans Le protestantisme (1987: 123-125) ; l'article "Le protestantisme " par Jean Baubérot dans Encyclopédie des religions (2005 : 295-307); l'article " Mission » par Klauspeter Blaser (2006), ibid et l'article d'André Roux, « Les missions protestantes » (Roux, 1972) et du même auteur Missions des églises, mission de l'Église (Roux, 1984). 
Les missionnaires essaiment de par le monde : Tahiti (Société missionnaire de Londres), Antilles et Afrique du Sud (missionnaires moraves)... Pour les Églises évangéliques issues des réveils (baptistes, méthodistes) : étendre le Royaume, c'est collaborer à l'œuvre de Dieu, répandre la parole divine, faire connaître Jésus, témoigner de sa miséricorde et de sa bonté. L'œuvre missionnaire s'investit alors dans la tâche d'évangéliser tous les «peuples » sur terre, de s'élancer à travers le monde.

Le XIX ${ }^{\mathrm{e}}$ siècle est donc le "grand siècle ${ }^{7}$ missionnaire. C'est au cours de ce siècle que les rapports entre mission et colonisation furent les plus étroits, étroitement complexes et partiellement conflictuels (Prudhomme, 2005) ${ }^{8}$. Des routes s'ouvrent, colons, marchands et missionnaires s'y engagent, leurs intérêts divergent ou convergent, c'est selon. À la faveur des conquêtes et des découpages de territoire (voir le Congrès de Berlin de 1885), les missions s'installent le plus souvent dans les colonies de leur nation-mère, comme l'écrit Claude Prudhomme " les réussites les plus spectaculaires des missions à partir de la découverte des Amériques en 1492 ont eu pour cadre les aires géographiques colonisées par l'Europe occidentale. À défaut d'être une condition suffisante, (...), la colonisation semble devenue depuis le $\mathrm{XVI}^{\mathrm{e}}$ siècle une condition quasi nécessaire à la christianisation, ce que tendent à confirmer les succès à l'époque moderne dans les Amériques et aux Philippines, puis à l'époque contemporaine dans une grande partie de l'Afrique noire et en Océanie » (Prudhomme, 2004 : 8-9). C'est dans ce contexte que le terme de mission se met à renvoyer à un territoire, on assiste donc à une territorialisation de la mission qui conduit à différencier la mission intérieure (action d'évangélisation dans les pays chrétiens) de la mission extérieure (action d'évangélisation dirigée vers les infidèles dans des pays lointains, le plus souvent des colonies). C'est donc au XIX siècle que s'élaborent des cartes des pays de mission, des cartes du territoire missionnaire, là ou des hommes et des femmes s'activent à répandre la Bonne nouvelle. Les missions catholiques ont été plus enclines à cartographier leurs implantations sans doute parce que les catholiques ont une "vision plus statique d'une Église aux structures bien définies (...). [L]es missionnaires, en quelque pays qu'ils se présentent, n'ont autre chose à faire qu'à la planter, toute faite, en communautés groupées sous la juridiction de Rome" (Roux, 1984 : 79), alors que les protestants ont " une conception plus dynamique : des missionnaires appartenant à des Églises diverses partent pour proclamer la Parole de Dieu, parole créatrice qui donnera naissance, par la conversion de "personnes", à des Églises locales appelées à être d'emblée membres de plein exercice du Corps du Christ, et dont on professe que l'Esprit les conduira à découvrir les structures qui seront pour elles les meilleures » (ibid. : 79).

7. L'expression est de Kenneth Scott Latourette, historien des missions.

8. Les interférences sont nombreuses entre mouvements de colonisation et actions missionnaires, un jeu de complicités et d'accusations. 
L'éclatement premier de l'entreprise missionnaire protestante et la rapide indigénisation des structures rendent difficiles la production de cartes des territoires missionnaires. Cependant, les composantes protestantes les plus structurées et institutionnalisées et fondamentalement les moins actives dans l'entreprise apostolique, comme les Anglicans qui s'appuient sur l'Empire britannique, élaborent à l'instar des catholiques, mais dans une moindre mesure, une cartographie des pays de mission.

Au cours du $\mathrm{XX}^{\mathrm{e}}$ siècle, les évangéliques américains s'imposent dans la course à l'évangélisation, grâce à la nébuleuse évangélique et aux pentecôtistes en particulier, et fournissent des contingents armés d'importants moyens et d'une ardeur évangélisatrice à toute épreuve (Ammerman, 2004 ; Noll, 2004). Mais, face « au morcellement confessionnel ", les missions protestantes ont peu à peu ressenti le besoin de se rassembler afin d'unir leurs efforts : en 1910, a lieu la Conférence missionnaire d'Édimbourg avec pour titre "Évangéliser le monde dans notre génération ". Cette conférence marque le point de départ du mouvement œcuménique moderne. Le Conseil CEcuménique des Églises (COE) apparaît sous sa forme institutionnelle le 23 août 1948. Dans le même temps, des alliances se constituent entre missions afin de coordonner leurs entreprises. Ainsi, du Mouvement de Lausanne qui naît à l'initiative de l'évangéliste américain Billy Graham, le "pape protestant " ${ }^{9}$ à la capacité de mobilisation extraordinaire. Au cours de juin 1974, se déroule à Lausanne (un lieu symbolique pour tous les protestants), un Congrès international de l'évangélisation mondiale qui prend fait et acte de la "décentration des changements sociaux " (Geschiere, Meyer, 2000 : 203), de la multipolarité des missions et de la pluralité de l'origine nationale des missionnaires. La contribution la plus importante du congrès de Lausanne est la rédaction d'une déclaration de foi trans-dénominationnelle qui peut se résumer dans le slogan "Toute l'Église offrant tout l'évangile à tout le monde ». C'est dans ce contexte d'œcuménisme, de structuration en réseau, de capitalisation des ressources en réponse à la globalisation rapide du religieux et en vue d'organiser une stratégie d'évangélisation totale que le spiritual mapping voit le jour.

\section{Aux origines du spiritual mapping}

\section{Spiritual warfare et inscription territoriale}

Les promoteurs du spiritual mapping s'inscrivent dans la vaste réflexion théologique, missiologique et eschatologique qui s'engage à la fin des années 1980 et au début des années 1990 au sein du milieu évangélique américain en particulier. Les chercheurs s'accordent aujourd'hui pour parler d'une nouvelle phase

9. L'expression est de Sébastien Fath (2002). 
dans le développement de la mouvance évangélique, certains parlent de néopentecôtisme (Martin, 1990), d'autres, comme Harvey Cox (1995) ou Sébastien Fath, préfèrent le terme de charismatisme ou de pentecôtisme de troisième vague. À la différence des précédentes vagues, cette troisième vague insisterait davantage, selon S. Fath, sur quatre points : 1. le territoire comme mode opératoire d'action spirituelle; 2 . la guerre spirituelle (spiritual warfare) comme entreprise d'évangélisation totale; 3 . l'évangile comme accès au pouvoir et à la richesse, c'est le power evangelism ; 4 . le prophétisme et le charisme des leaders chrétiens (Fath, 2007). Ce sont ces deux premiers points, territoire et guerre spirituelle auxquels nous allons nous intéresser.

Donner sens et agir peuvent caractériser le spiritual warfare. Entrer en guerre pour Dieu, c'est adopter une attitude active (pro-active selon le terme anglais) dans sa foi (un tel activisme fait résonance à cette montée des activismes militants toutes identités confondues et ces réveils de fierté que l'on trouve dans ce qu'il est convenu d'appeler les minorités). L'expression se réfère à plusieurs citations provenant de lettres de Paul (Éph 6,10-18 ; 2 Cor 10,3-5); aux Éphésiens, il écrit "C'est pourquoi prenez toutes les armes de Dieu, afin de pouvoir résister dans le mauvais jour " (Éph 6,13). Ces armes, précise Paul, sont des armes spirituelles et, parmi elles, le chrétien peut se référer aux fruits de l'Esprit (Gal 5,22-23). Devenir un guerrier pour Dieu suppose un ennemi, une puissance à combattre. "Car nous n'avons pas à lutter contre la chair et le sang, mais contre les dominations, contre les autorités, contre les princes de ce monde de ténèbres, contre les esprits méchants dans les lieux célestes » (Éph 6,12). Si des forces travaillent à contrecarrer les plans de Dieu (et l'on voit comment l'état du monde peut être lu à l'aune de cette doctrine), le chrétien doit s'engager dans une lutte incessante par les armes de la prière, de la rectitude, de l'activisme prosélyte... Comme dans toute guerre, l'élaboration de stratégies implique une perception de l'espace et un rapport symbolique et affectif à un territoire. L'affirmation d'un territoire ou du moins sa symbolisation participe d'une action revendicatrice dans un monde globalisé. La marche pour Jésus en est un exemple.

Les marches pour Jésus (March for Jesus), débutent en Angleterre dans les années 1980 quand des Églises locales décident de prendre la rue, de servir Dieu en dehors des temples. En mai 1987, plusieurs organisations chrétiennes se rassemblent pour une marche de prière et de louange à la gloire de Jésus à travers les rues de Londres. Dans les années 1990, le mouvement, qui s'est établi entre temps aux États-Unis, gagne de nombreux pays à travers le monde. Les chrétiens désirent à travers cette marche exprimer leur foi, la rendre visible et présente dans le monde. Sans doute, peut-on voir un mouvement d'affirmation positive (la marche se déroule sur les grandes avenues et places, elle se veut festive et musicale) à l'égard de l'extérieur. Il y a comme une volonté de se faire voir au moment où l'on croyait la religion disparue des mégapoles. Les rues des villes, prises d'assaut par le commerce, la marchandisation, le règne de l'argent, ont 
participé de ce mouvement de sécularisation, de démagification où les relations ne sont, souvent, plus basées que sur la confiance dans une monnaie. Les espaces urbains s'ouvrent à la diversité et en même temps à leur indifférenciation par les flux urbains (circulation piétonnière et motorisée, foule, signalisation où chacun se réfère à un même code et de mêmes règles - de la route, du savoir-vivre...). Et tandis que les protestants se fermaient à l'extérieur au cours de leur rituel (service dominical et études bibliques dans le temple), des religions et spiritualités investissaient l'espace public comme un espace d'action (que l'on pense au mouvement "Hare Krishna " très présent en Angleterre et dans les rues de Londres) ou avaient une tradition déjà ancrée de publicisation (les processions catholiques, par exemple). En réaction au double mouvement de sécularisation de l'espace urbain (sentiment de perte de contrôle de cet espace) et de l'éclosion de mouvements religieux publicisant leur croyance, les protestants ont dû se soumettre au « devoir d'exposition" (Joseph, 1998 : chap. I). Se rendre visible (par temps de concurrence exacerbée entre religion, marchandisation, publicité) signifie prendre possession de manière symbolique d'un territoire dominé par des règles et des valeurs divergentes (Fer, 2007). L'espace urbain, le territoire des villes, mais aussi des campagnes, deviennent des enjeux religieux à partir du moment où ils sont inscrits dans le vaste mouvement de flux mondialisés. D'où l'ambiguïté de la globalisation, pour exister, il faut pouvoir s'enraciner dans un territoire (et pas seulement agir sur la dimension transnationale) afin de le rendre "sien » à mesure qu'il se pluralise dans des "nous ". Cette action d'affirmation et de visibilisation sur un territoire participe d'une nouvelle expression religieuse territoriale qui a partie liée avec le spiritual mapping.

\section{Spiritual mapping, deux versions}

Le spiritual mapping peut être défini de manière englobante comme l'élaboration contemporaine de cartes ordonnançant le monde selon des composantes religieuses, sociales, culturelles, etc., en vue d'organiser et de faciliter le travail missionnaire évangélique. Mais, si l'on y regarde de plus près, on s'aperçoit que le spiritual mapping est loin d'être uniforme. J'ai repéré, pour ma part, deux grandes versions qui se partageraient le champ de la cartographie spirituelle évangélique et que j'ai, faute de mieux, dénommées :

- le spiritual mapping "surnaturel », qui s'inspire de Wagner, et conçoit la lutte en terme de forces surnaturelles dépassant les hommes (modèle d'une modernité de la science-fiction, de la fantasy);

- le spiritual mapping " réaliste ", qui se voudrait comme un pur miroir, une pure institution de la réalité, cette cartographie n'est pas dénuée de prétention scientifique et se révèle le creuset d'une géographie technicisante (modèle d'une modernité des sciences).

Je voudrais donner un rapide aperçu du spiritual mapping version "surnaturelle " car c'est à partir de cette version, et dans sa confrontation, que la 
version "réaliste ", celle qui nous intéresse, s'est développée. L'expression de spiritual mapping a été forgée, en 1990, par George Otis sous l'influence de John Dowson qui avait publié, en 1989, Taking our Cities for God. How to Break Spiritual Strongholds, tandis que trois ans auparavant, Frank E. Peretti, écrivain de best-sellers chrétiens, publiait un livre de fiction au titre évocateur This Present Darkness (1986). Peretti y raconte la lutte des anges contre les démons (une « secte " New Age) pour le contrôle de la petite ville d'Ashton dans une narration relevant des codes de la fantasy. Otis explicite le spiritual mapping en 1991 dans un ouvrage intitule The Last of the Giants. Lifting the Veil on Islam and the End Time : il y fait état de trois forces (le matérialisme, l'hindouisme et l'islam) qui contrecarrent le plan de Dieu (la rédemption de toutes les nations). Ces forces agissent d'abord sur le plan surnaturel : il faut donc apprendre le "langage et les secrets de la dimension spirituelle " (1991: 80 ; la traduction est la nôtre) mais également saisir les références historiques, économiques, institutionnelles de l'aire où l'on veut agir (dimension pseudo scientifique moderne) afin de les décoder (saisir les forces démoniaques en présence) et de les inscrire sur des cartes utiles à l'action spirituelle (marche de prière, évangélisation localisée...).

George Otis, peut être considéré comme une figure archétypique de la troisième vague à l'œuvre dans le pentecôtisme : il s'impose comme un leader charismatique d'importance, il fut un des acteurs majeurs du Mouvement de Lausanne, il joue avec brio des logiques de la société marchande (écrivain de best-sellers, fondateur d'une organisation chrétienne transnationale puissante et active, le Sentinel Group), il prône le power evangelism en donnant au croyant des clés interprétatives basées sur une logique explicatrice manichéenne et simplifiée à l'extrême pour s'orienter dans un monde difficilement déchiffrable. Sa thèse a été reprise par Peter Wagner qui va devenir l'un des grands promoteurs du spiritual warfare et de la technique du spiritual mapping. Wagner a un profil de leader comparable à celui d'Otis. Écrivain de best-sellers, il pénètre plus encore dans le domaine des esprits et sa position participe d'un ré-enchantement du monde que l'on avait, trop tôt, donné comme disparu. Pour Wagner, la guerre spirituelle se situe clairement sur le front démoniaque ${ }^{10}$. Il élabore le principe d'un strategic-level spiritual warfare (SLSW) dans lequel il développe la notion de corporate exorcism. Il ne s'agit plus de délivrer un individu des forces du mal, mais de rendre à sa liberté un territoire plus ou moins important. Dans ce cas, le spiritual mapping tente d'identifier le type d'esprit maléfique associé à un territoire en vue de le lier et de l'éloigner. Ces esprits démoniaques agissant sur un territoire, le contrôlant, l'asservissant, et le transformant en forteresse sont appelés "territorial spirits». Wagner différencie trois niveaux de bataille: 1. groundlevel spiritual warfare se réfère aux ministères de la délivrance et de l'exorcisme ; 2. occult-level spiritual warfare tente de se confronter aux activités comme le

10. Voir sa série "The prayer warrior" qui comprend actuellement sept tomes, et en particulier (Wagner, 1991 et 1993). 
satanisme, la franc-maçonnerie ou le chamanisme; 3. strategic-level spiritual warfare a pour but de localiser, d'identifier et de faire disparaître les formes démoniaques du haut de la hiérarchie que sont les "esprits territoriaux" qui " rendent esclaves" des "peuples", des «nations", des «villes "... Avec le SLSW, il s'agit donc de discerner les tendances spirituelles (qualifiées de démoniaques à partir du moment où elles ne sont pas chrétiennes) qui parcourent un territoire, un réseau, une institution. La cartographie s'élabore à partir de recherches historiques, économiques et sociales qui se focalisent sur les traumatismes historiques, les conflits idéologiques, les "idolâtries "... La carte repère les lignes de force (des informaticiens acquis au SLSW ont mis en place des programmes informatiques complexes de cartographie), les clefs d'entrée des esprits sataniques. Les actions à entreprendre, qui se structurent selon les tendances dégagées, consistent alors en prières, en corporate exorcism, en implantation d'églises... Des actions de SLSW ont ainsi eu lieu contre les esprits de luxure qui domineraient le district Red light d'Amsterdam ou sur les esprits de sorcellerie de la Nouvelle-Orléans. Le système de Wagner a trouvé une seconde vie dans l'hémisphère Sud où il est utilisé dans les pays à traditions religieuses polythéistes.

Les thèses de la SLSW ont été reprises par les dénominations pentecôtistes, les Églises charismatiques ou prophétiques, mais le point de vue de Wagner est considéré par une large frange de protestants (Églises historiques et une partie des évangéliques) au mieux comme abusif, au pire comme aberrant (aucun fondement biblique). Le jeu du « qui croit vrai » ou de l'auto-désignation orthodoxe et du renvoi de l'autre (Église, mouvement) dans l'hétérodoxie s'élabore autour de ces discussions doctrinales. Mais, nous allons le voir, les évangéliques opposés à la SLSW ne font pas moins usage du spiritual mapping en le concevant comme un outil à prétention scientifique afin de repérer les grandes tendances religieuses, spirituelles, linguistiques, économiques d'une zone d'action.

\section{Cibler, nommer, cartographier}

\section{Viabilité, coordination, objectifs}

Devant la complexification du monde, des organisations chrétiennes basées principalement aux États-Unis - malgré les changements en cours qui ouvrent la pratique missionnaire à des natifs du Sud ${ }^{11}$, les évangéliques américains restent

11. Si les États-Unis sont toujours le premier pays à envoyer le plus grand nombre de missionnaires (protestants, indépendants et anglicans) à travers le monde, la Corée du Sud précède le Royaume-Uni. Mais, si l'on considère à présent le nombre de missionnaires envoyés à l'étranger par rapport au nombre de chrétiens de même origine nationale, c'est la Mongolie qui arrive en tête avec un missionnaire pour mille deux cent vingt-deux chrétiens, suivi du Liban et Singapour. Les États-Unis ne figurent pas dans ce peloton de tête, seule l'Espagne (septième position) représente les pays occidentaux. 
très mobilisés, actifs et puissants dans l'action missionnaire grâce, entre autres, à une mise en œuvre efficace de relations et de réseaux (capacité de mobilisation très large dans les domaines politiques, économiques, people), de ressources humaines, technologiques, et financières - ont opté pour les méthodes de marketing qui ont révélé leur efficacité dans le domaine de l'économie. Rendre cohérentes et efficaces leurs actions doit passer, en premier lieu, par l'élaboration de stratégies pragmatiques. Car si les évangéliques font front contre la modernité, ils n'en épousent pas moins certaines de ses composantes, et se révèlent même des produits de cette modernité ${ }^{12}$.

Le US Center for World Mission, fondé en 1976 par des missionnaires américains, se donne justement pour tâche de définir les nouveaux enjeux missionnaires : faciliter le travail des missionnaires par des services adaptés, mobiliser les ressources en étudiant la cartographie des "peuples " qui n'ont pas encore été évangélisés, en rendant compte des avancées et en s'assurant de l'exécution d'un projet qui devait se finaliser en l'an 2000 «Une Église pour chaque "peuple”, l'évangile pour chaque personne". De nombreux ministères lui sont rattachés, les collaborations sont pléthores et sa structure se décompose en quatre branches: - stratégie : rechercher et savoir quels sont les peuples non encore évangélisés, trouver les outils opérants et efficaces pour parvenir à leur évangélisation en implantant des églises viables et dirigées par des indigènes ;

- mobilisation : rassembler les énergies missionnaires et s'assurer de leur complémentarité ;

- formation : le missionnaire doit être "formé stratégiquement afin qu'il soit opératoire de la meilleure manière possible dans les lieux les plus critiques ${ }^{13}$; - services : mettre à disposition des missionnaires, des ressources et des données.

L'US Center for World Mission fait état, sur son site, de deux optiques missionnaires qui se seraient succédées au cours des dernières décennies et qui montrent une petite réforme interne à la pratique missionnaire (une réforme missionnaire qui accompagne et rend compte des transformations en cours dans la sphère religieuse évangélique). Alors que dans les années 1970, certains missionnaires appelaient à un moratoire sur les missions (la tâche missionnaire était accomplie et les indigènes allaient entreprendre leur propre révolution évangélisatrice), les années 1990-2000 ont, au contraire, souligné les besoins nouveaux en évoquant des populations qui n'avaient pas encore été touchées par l'évangile, en réaffirmant la nécessité d'une vaste entreprise missionnaire critique quant à ses agissements (besoin d'implanter des églises dirigées par des autochtones dans le respect des cultures, réflexion sur l'inculturation...) dans un contexte global de flux et de conflits, d'affirmations identitaires religieuses. Cette nouvelle optique missionnaire permet de légitimer la présence missionnaire et permet également de répondre aux critiques dirigées contre les missions perçues comme agent d'assimilation.

12. Selon Danièle Hervieu-Léger, la modernité même engendre la religion (1986).

13. http://www.uscwm.org/, page division - mobilization, traduction de l'auteur. 
76 - ARCHIVES DE SCIENCES SOCIALES DES RELIGIONS

\section{«Tout lieu que foulera la plante de votre pied, je vous le donne » (Jos 1,3)}

L'un des ministères affiliés à l'US Center for World Mission est le Joshua Project $(\mathrm{JP})^{14}$. Le JP naît en 1995 de la nécessité de déterminer avec précision la nouvelle cible de l'évangélisation, son slogan "Apporter des définitions à la tâche inachevée ". Il se fixe pour mission "d'identifier et d'éclairer les peuples à travers le monde qui ont été le moins exposés à l'évangile ». L'idée du JP est de transférer la fameuse stratégie marketing de Coca-Cola ( Une canette de Coca Cola dans la main de chaque personne sur la terre ») à l'entreprise missionnaire. Pour ce faire, il tente de répondre à trois questions : 1. "Quels sont les groupes ethniques à travers le monde ?"; 2. "Quels groupes ont besoin en leur sein d'une première église "; 3. "Quelles sont les ressources valables pour parvenir auprès de ces populations qui n'ont pas encore été touchées par l'évangile ? " ${ }^{15}$. Ces trois questions impliquent, en premier lieu, de déterminer une cible d'action. La cible fondamentale se développe à partir de la notion de unbelievers (non croyants). Par unbeliever, il faut entendre, selon ces organisations, une personne qui n'a pas accepté le Christ (au fondement de la foi évangélique on trouve une vérité et une seule). Seul est croyant (believer) celui qui croit en Christ Rédempteur. Parmi ces unbelievers, s'est imposé un groupe à part qui constitue la nouvelle pierre philosophale de l'évangélisation: il s'agit des unreached et des leastreached people group (ULPG). Comme le terme anglais l'indique, les ULPG sont ceux qui n'ont pas encore été touchés (ou été peu touchés) par l'évangile, ceux qui ne savent rien (ou peu) de la Bible, du Christ, de l'Église, ce sont les «peuples» ayant une communauté indigène chrétienne inexistante avec des ressources humaines et matérielles insuffisantes pour mener à bien l'évangélisation. Les organisations chrétiennes entendent par people group ${ }^{16}$ " le plus large des groupes à l'intérieur duquel l'évangile peut être répandu à travers un mouvement d'implantation d'église sans que la propagation ne rencontre de barrières à la compréhension et à l'acceptation " ${ }^{17}$. L'utilisation de ce terme se réfère à Gen 12,1-3, Dieu veut se faire connaître de tous les "peuples " (cf. également Marc 13-10 et Ap 5,9). La barrière à la compréhension étant la principale des deux, le critère linguistique domine largement la délimitation et la définition des « peuples » du monde. C'est pourquoi le Joshua Project se réfère à l'encyclopédie Ethnologue. Languages of the World (Gordon, 2005) ${ }^{18}$ orchestrée par le Summer of Institute Linguistic

14. Joshua Project prend pour nom un personnage biblique, Josué. Le livre de Josué est un souffle d'espoir après l'exode d'Égypte. Josué mène les campagnes militaires en vue de reconquérir le pays promis (Jos 1,6) et s'en remet à Dieu dans une confiance absolue. Le livre se lit comme un ouvrage de géographie, les noms de lieux font référence aux différentes batailles et à la répartition des terres entre les tribus d'Israël.

15. http://www.joshuaproject.net/joshua-project.php.

16. Que je traduirai pour faciliter la lecture par "peuple " ou "groupe ethnique ".

17. http://www.joshuaproject.net/definitions.php\#peoplegroup, la traduction est la nôtre.

18. Version internet : http://www.ethnologue.com/Wycliffe. 
International. Cette organisation chrétienne américaine se propose de recenser et de connaître les langues minoritaires non écrites à travers le monde dans le but de traduire la Bible. Son fondateur, le missionnaire américain William Cameron Townsend (1896-1982), joue du slogan «pour chaque homme, chaque femme, chaque enfant, une Bible dans leur langue».

Le Joshua Project se présente donc sous la forme d'une base de données extrêmement riche offrant une liste qui se veut exhaustive de tous les " peuples " recensés dans le monde ${ }^{19}$. Il propose une géographie qui combine une cartographie, une recension des populations, des mœurs, des langues, des religions, des politiques, à la manière d'une ethnologie monographique à l'ancienne mode. Le Joshua Project recense 15972 "groupes ethniques » et nationaux à travers le monde, 6599 sont enregistrés en tant que groupes unreached/least-reached totalisant plus de deux milliards d'individus. Cette entreprise monumentale qu'est le JP consacre la diversité dans l'humanité une de la création divine. Pour les évangéliques, la diversité, résultat de la tour de Babel, doit être ramenée à l'unité dans la croyance du Dieu unique. Ce qui fait la spécificité de JP, et lui vaut son statut de référence mondiale pour les missions de toutes nationalités et dénominations, est la cartographie. Il a recours, pour le savoir cartographique, à l'organisation chrétienne Global Mapping International (GMI). Fondé en 1983 sur le campus de US Center for World Mission, le GMI se propose de fournir aux missions et Églises des cartes des «terrains de moisson 》 (harvest fields). Pour ce faire, il utilise le Système d'Information Géographique (SIG). Il s'agit de logiciels informatiques qui permettent d'établir des cartes complexes modulant à la fois des données géographiques (délimitation d'un espace : une région, une ville...), des données descriptives (les attributs que l'on veut voir apparaitre sur la carte du type politique, démographique, religieux) et des données graphiques (choix des couleurs....). L'élaboration de cartes par le GMI, à la demande des missionnaires, doit permettre de proposer des ressources actualisées sur les terrains d'action de ces missionnaires chrétiens et fournir des données géographiques d'importance dans la constitution d'une stratégie globale d'évangélisation.

\section{Précautions de lecture}

S'intéresser à la carte comme objet d'étude c'est se poser des questions essentielles : qui sont les mapmakers (ceux qui fabriquent les cartes)? Qui est le commanditaire ? À qui sont adressées les cartes ? En vue de quels usages? Des interrogations qui font dire à Patrick Poncet que la carte est un « objet en relation » (2007), et que le discours qu'elle porte est relatif, relatif par rapport à ses

19. Dans une perspective semblable (des données sur les "peuples " non encore évangélisés pour une meilleure évangélisation), on peut se référer également à Ethnê (http://ethne.net/) et Mission to unreached peoples qui focalise ses activités missionnaires sur l'Asie et le MoyenOrient (http://www.mup.org/index.php). 
concepteurs et par rapport au public auquel elle s'adresse. La carte n'a pas prétention à la vérité, la reproduction qu'elle en fait présuppose un choix de critères (d'échelle, de localisation - quel élément central, quel autre en périphérie...) pour lui faire dire ce que l'on veut lui faire dire et qui ne sera peut-être pas forcément lu de cette manière. En avançant sous le masque d'une «science apparemment neutre » (Bailly, Gould, 1995: 72), la carte campe sur une image d'autorité et oblige à la confiance, ce qui a, sans doute, séduit les missionnaires chrétiens en mal de légitimation et de crédibilité dans un contexte de sécularisation croissante et de credo technologico-numérique.

\section{Ouvrons la fenêtre $10 / 40$}

Une notion géographique a peu à peu émergé dans la réflexion missionnaire : il s'agit de la notion de 10/40 Window, forgée au début des années 1990, par le stratège chrétien Luis Bush. La 10/40 Window est autrement appelée resistant belt (la " ceinture de résistance ", le terme de ceinture est couramment employé en géographie, on le retrouve, par exemple, dans la notion de Bible Belt pour désigner les États du sud-est des États-Unis dans lesquels se trouvent le plus grand nombre d'évangéliques et dont la politique est fortement empreinte de religion). Ouvrons une parenthèse. Se fait jour, dans ces organisations évangéliques, un langage indigène très technique usant d'abréviations où le Nouveau Testament se résume en NT, les peuples non encore évangélisés en ULPG, les stratégies de guerre spirituelle en SLSW. De tels sigles assurent la cohésion identitaire du mouvement par un langage commun et en même temps soumet ce langage à des exigences non plus tant théologiques que pseudo scientifiques, un langage de technicité qui a cours dans la société américaine (l'abréviation est une forme de langage à grande vitesse propre à nos sociétés modernes) et qui s'en vient tout droit de l'économie et du marketing, un langage de moyens, de finalités, de résultats saupoudré de références bibliques.

Cette zone géographique, définie par un rectangle partant de l'Afrique de l'Ouest passant par le Moyen-Orient pour se terminer dans l'Est de l'Asie, du dixième au quarantième parallèles de l'hémisphère Nord, serait le " cœur du cœur » des groupes non touchés ou peu touchés par l'évangile (UPG). Cette aire comprendrait cinquante-neuf pays et un total de quatre milliards d'habitants. La carte que nous présentons ci-dessous fait partie d'un dossier du magazine Time titré "Les chrétiens devraient-ils convertir les musulmans ? " (Time, 2003). Plusieurs articles traitent des missions dans les pays musulmans et soulignent les persécutions dont sont victimes les missionnaires. La carte n'est pas du Time, le magazine la tire du World Christian Trends, source majeure et incontestée dans le domaine des statistiques sur les chrétiens dans le monde. Sans doute parce que nombre d'esprits américains se fixent sur les pays musulmans, Time ne dit rien des autres pays où pourtant les missionnaires sont présents en plus grand nombre. 


\section{0/40 Window et présence missionnaire}

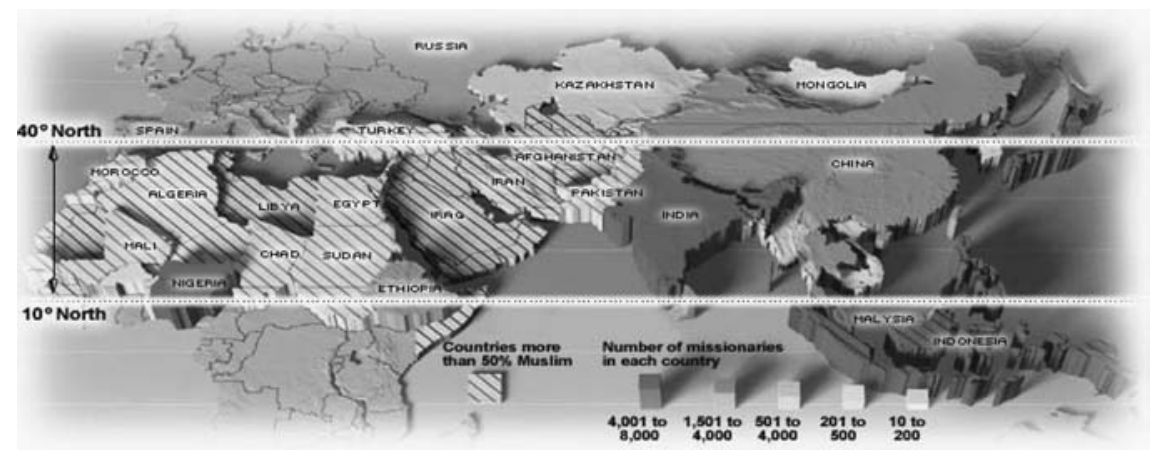

Source : Time Magazine 30 juin 2003 Issue

Sept raisons sont évoquées par les missionnaires évangéliques pour justifier leur investissement dans cette zone : 1. la plus essentielle, à leurs yeux, est celle de la signification biblique de cette zone qui comprend la Palestine et les premiers pays évangélisés avant l'expansion de l'islam, c'est en ces lieux que le premier couple aurait trouvé place. À l'unité première aurait succédé, à cause de l'orgueil de l'homme (Gen 11,1-9), une multitude de langues et de nations rendant difficile l'évangélisation. C'est aussi cette terre que Dieu promet à son peuple élu dans l'Ancien Testament, c'est le lieu de l'alliance accomplie (la terre qui a vu naître, vivre et prêcher le fils de Dieu). C'est enfin le lieu où, dans les derniers temps, le rassemblement de tous les juifs sur la terre d'Israël annoncera la seconde venue du Christ (on voit comment les chrétiens s'approprient cette terre par références bibliques et, dans une dramatisation élaborée, réveillent l'intérêt missionnaire pour ce lieu soit disant déserté par les chrétiens) ${ }^{20} ; 2$. la région la moins évangélisée ; 3. la domination de "trois blocs religieux »: islam, bouddhisme, hindouisme ; 4. des pays pauvres ; 5 . des groupes ethnolinguistiques sans traduction partielle ni traduction totale de la Parole de Dieu ; 6. des mégapoles peu touchées par l'évangile ; 7. une forteresse de Satan ${ }^{21}$.

Mais, ce qui apparaît d'abord, c'est l'intérêt des organisations évangéliques pour une zone relevant de la géographie obsessionnelle de la politique étrangère américaine. Les pays de l'Axe du mal y sont, pour la plupart, situés et l'on sait combien la politique étrangère du président américain George Bush Jr. trouve

20. Il est à noter que la sacralisation de l'aire Palestine-Israël et de sa centralité dans l'eschatologie chrétienne doit être prise en compte pour mieux comprendre la géopolitique américaine. Le sionisme développé avec force par certains chrétiens américains d'obédience fondamentaliste s'explique dans cette insertion de la Palestine dans l'histoire chrétienne passée et à venir. Voir Mearsheimer et Walt (2007).

21. Ces raisons ont été développées, en particulier, par le réseau Ad2000 et Beyond Movement, http://www.ad2000.org/1040broc.htm. 
ses inspirateurs dans l'aile fondamentaliste chrétienne dont les préoccupations sont souvent relayées, avec modération et de manière sélective, par les évangéliques piétistes ou pentecôtistes. Or, la notion de10/40 Window se développe dans les années 1990, au moment de la guerre du Golfe et prend de l'ampleur dans les années 2000, alors que les États-Unis élaborent une politique ferme et intrusive en matière d'anti-terrorisme dans les pays de l'Afrique de l'Est, du Moyen-Orient... L'amalgame islam-islamisme-terrorisme rend l'action missionnaire dans la région comme une urgence nationale et internationale. Les organisations chrétiennes sont d'ailleurs les premières à soutenir les soldats en guerre. Et Peter Wagner qui, je le rappelle, est un des promoteurs de la version « surnaturelle » de la spiritual mapping, invite les missionnaires à se concentrer sur une nouvelle fenêtre plus restreinte qu'il nomme 9/11 Window en référence aux attentats du 11 septembre 2001 contre les États-Unis et qui inclut tous les pays à population musulmane majoritaire.

Cette carte a été reprise par le site Internet du Joshua Project et s'adresse donc cette fois à un public acquis. Tandis que Time accompagnait la carte d'une question et laissait la réponse au lecteur, JP présuppose qu'il est d'une grande urgence de convertir la zone 10/40 Window. Par cette vue partielle de la réalité, plusieurs continents rassemblés excluant les Amériques et le Pacifique et par un encadré conduisant l'attention du lecteur, JP cherche à insister sur la localisation des interventions. La répartition par couleurs permet au lecteur chrétien de visualiser avec facilité et rapidité les aires selon les besoins missionnaires. Face à cette fenêtre il se dégage comme une injonction: celle de l'évangélisation. La carte relève d'un procès actif : "stimuler l'intérêt des missionnaires pour les terres “hostiles ». La carte s'avère être un puissant instrument de persuasion. Elle est importante dans la maîtrise et dans l'appropriation virtuelle d'un territoire. Elle peut alors précéder le territoire, le fantasmer en un "espace satanique " et le rêver en une " terre chrétienne".

\section{0/40 Window et « blocs religeux »}

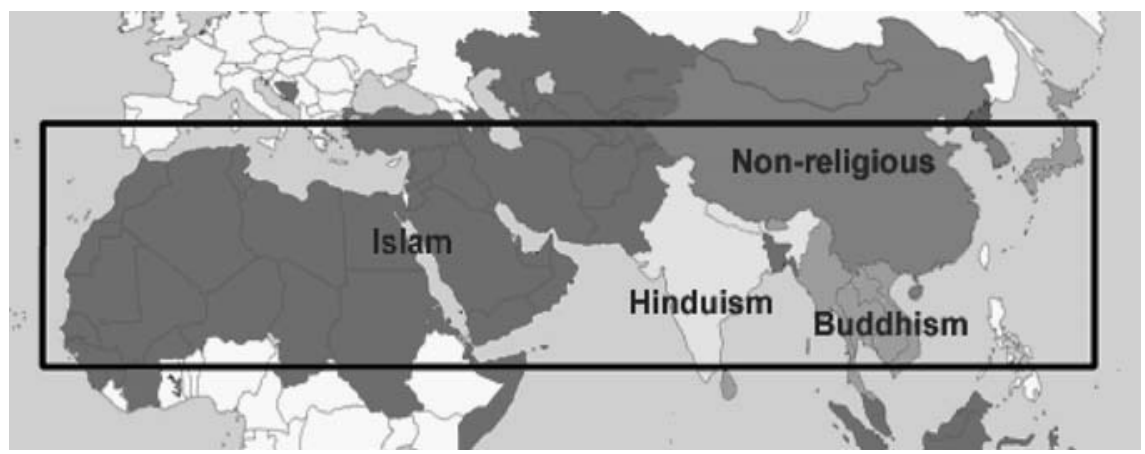

Source : Joshua Project et GMI, http://www.joshuaproject.net/10-40-window.php 
La carte est aussi une géographie théâtralisée, comme ci-dessus, dans ce jeu dramatisant d'homogénéité et d'hétérogénéité produit par les couleurs : un «bloc" islam imposant, une Chine (à laquelle on adjoint la Mongolie) non religieuse (Qu'entendre par ce terme? Qu'entendra celui qui visualise ?), une absence de la religion juive (on croit discerner un État blanc entouré d'un vaste îlot vert musulman), une annihilation des minorités religieuses, des spiritualités. L'espace des continents est offert aux opérations réductrices de la graphie (constitution de "blocs»), une graphie qui s'institue "en lieu de l'autre " (Certeau, 1975 : 216). Il s'agit donc de nommer pour mieux réduire la diversité religieuse et instituer l'autre dans son statut imposé de type religieux (les photos du site du Joshua Project jouent également ce rôle en présentant un homme ou une femme en avant-propos d'une description d'un "groupe ethnique»). Les processus cartographiques, écrivent Gould et Bailly, " consistent en actes délibérés, (...) en adaptations cognitives conformes aux valeurs et aux croyances dominantes (...). Ils fournissent les règles cachées d'un discours cartographique qui trouve son origine dans les géométries subliminales, les silences et les hiérarchies représentées sur les cartes» (Bailly, Gould, 1995 : 50). Par géométries subliminales, les deux auteurs entendent, les choix cartographiques opérés qui sont tus : centralité/périphérie, type de projection... Dans la carte, ce n'est pas l'espace réel qui apparaît mais une conception de l'espace et, en l'occurrence, un espace catégorisé de l'altérité religieuse qui s'expose comme un espace de potentialité, un espace d'un agir à concrétiser.

Le Joshua Project et le Global Mapping International s'appliquent à la fabrication d'une géographie missionnaire nouvelle élaborant un cadre de référence pragmatique en traçant des frontières selon des critères religieux binaires : believers/ unbelievers, evangelized/unevangelized et reached/unreached. Le monde est cartographié, balisé, chiffré selon les valeurs évangéliques et ouvre des espaces de conquête nouveaux, dits vierges (de présence chrétienne) qui s'offrent comme des eldorados missionnaires, de nouvelles frontières (fronts pionniers) à repousser (l'entreprise n'est pas dénuée de frissons : des «peuples" difficiles à atteindre, des mœurs étranges, des croyances sans doute rétives à l'évangélisation) réactivant l'imaginaire de la Frontière américaine, ce point de rencontre entre la civilisation et la sauvagerie (Turner, 1963). Le spiritual mapping peut donc être appréhendé comme une géographie axiologique et pragmatique déployant des significations religieuses, politiques et imaginaires. Axiologique parce que le choix des données descriptives et des données graphiques à l'origine de la carte est élaboré en fonction des valeurs évangéliques (conception chrétienne du bien et du mal sur laquelle sont greffées des préoccupations politiques, lesquelles se réfèrent à l'idée $\mathrm{du}$ « choc des civilisations »); pragmatique parce que la carte n'est pas une fin en soi mais un moyen, c'est-à-dire un outil d'action pour parvenir à une fin (des territoires à conquérir pour le Christ et au nom du Christ). 
82 - ARCHIVES DE SCIENCES SOCIALES DES RELIGIONS

Au regard de l'histoire esquissée à grands traits dans notre première partie, le spiritual mapping version " réaliste " s'inscrit en droite ligne dans cette " cartographie chrétienne » et dans la géographie humaine enseignée par les jésuites, qui furent sans doute les précurseurs d'une géographie et d'une cartographie à destination de l'entreprise missionnaire. Avec la géographie des jésuites, la version "réaliste» partage la volonté d'élaborer une connaissance scientifique des " peuples » à évangéliser loin des premières « fictions mixtes » de la cartographie médiévale. A contrario, le spiritual mapping version "surnaturelle " renoue à sa manière avec l'enchantement des mappemondes. Si la toile de fond est conventionnelle et scientifique dans sa norme (un planisphère à la géométrie euclidienne), elle est néanmoins le champ de bataille où combattent des forces surnaturelles, des anges et des démons, le Bien et le Mal. Les deux versions du spiritual mapping offrent cependant un nouveau visage de la «cartographie chrétienne " par rapport à leurs prédécesseurs : elles sont toutes deux l'expression d'une modernité religieuse. Elles s'élaborent à partir de techniques informatiques complexes, et tandis que la version " réaliste " s'inspire de la technicité du marketing et du management, la version "surnaturelle" trouve ses topoi dans la science-fiction. Le spiritual mapping active, dans une mise en espace normée (carte), des logiques de symbolisation et de territorialisation, de virtualités et d'anticipation anxiolytique et cathartique dans un monde globalisé supposé menaçant.

Nadège MÉZIÉ

Doctorante en anthropologie Paris 5 René Descartes

nadegemezie@yahoo.com

\section{Bibliographie}

AMMERMAN N.T., 2004, "Le congrégationalisme : repli contre-culturel ou tremplin vers la modernité ? ", in Fath S., (éd.), Le christianisme évangélique : un christianisme de conversion, Turnhout, Brepols, pp. 215-233.

BAILly Antoine, Gould Peter, 1995, Le pouvoir des cartes. Brian Harley et la cartographie, Paris, Anthropos/Economica.

Baubérot Jean, 2005, "Le protestantisme ", in Encyclopédie des religions, in Encyclopeedia Universalis, vol. 1, Paris, Promotion Presse Mediasat, pp. 295-307.

Baubérot Jean, Willaime Jean-Paul, 1987, « Missions », in Le protestantisme, Paris, M.A. Éditions, pp. 123-125.

BebBington Davi, 1989, Evangelicalism in Modern Britain. A History from the 1730s to the 1980s, Londres-New York, Routledge.

Blaser K., 2006, "Mission ", in Encyclopédie du protestantisme, Paris/Genève, PUFLabor et Fides, pp. 906-918. 
Bosch David J., 1995, Dynamique de la mission chrétienne. Histoire et avenir des modèles missionnaires, Paris-Lomé-Genève, Éditions Karthala-Haho-Labor et Fides.

Bouloux Nathalie, 2006, "Encore quelque réflexions sur l'usage des cartes par Pétrarque ", Quaderni d'Italià, 11 (Barcelone), pp. 313-326.

BouYer Marc, Duviols Jean-Paul, 1992, Le théâtre du Nouveau Monde. Les grands voyages de Théodore de Bry, Paris, Gallimard.

CerTeau Michel de, 1975, "Ethno-graphie. L'oralité, ou l'espace de l'autre : Léry ", in L'écriture de l'histoire, Paris, Gallimard, pp. 215-248.

Cox H., 1995, Retour de Dieu. Voyage en pays pentecôtiste, Paris, Desclée de Brouwer. DAINVILle François de, 1969, La géographie des humanistes, Genève, Slatkine reprints.

Dilke O.A.W., 1987, " Cartography in the Byzantine Empire », in Harley J.B., Woodward D., (eds.), The History of cartography, vol. 1, Chicago, University of Chicago Press, pp.258-275.

FATH Sébastien, 2002, Billy Graham. Pape protestant? Paris, Albin Michel.

-, 2007, "Les charismatiques de troisième vague, c'est quoi ? ", in http://blogdesebastienfath. hautetfort.com/archive/2007/05/03/les-charismatiques-troisieme-vague-c-est-quoi2.html\#more.

FER Yannick, 2007, «Pentecôtisme et modernité urbaine : entre déterritorialisation des identités et réinvestissement symbolique de l'espace urbain ", Social Compass, 54-2, pp. 201-210.

GesChiERE Peter et MeYer Birgit, 2000, «La réponse aux critiques de P. Geschiere et B. Meyer ", Politique Africaine, 78, pp. 201-207.

GoRDON Raymond Jr., (ed.), 2005, Ethnologue: Languages of the World, Dallas, SIL International.

Hervieu-LÉger Danièle, Vers un nouveau christianisme? Paris, Éditions du Cerf, 1986.

JosepH I., 1998, La ville sans qualités, La Tour d'Aigues, Éditions de l'Aube.

KILANI Mondher, 2000, L'invention de l'autre. Essai sur le discours anthropologique, Lausanne, Payot.

LeCoQ D., 1989, "La "mappemonde” du De Arca Noe Mystica de Hugues de SaintVictor (1128-1129) ", in Pelletier M., (éd.), Géographie du monde au Moyen Âge et à la Renaissance, Paris, Éditions du CTHS, pp. 9-31.

Leplay Michel, 2004, Les protestantismes, Paris, Armand Colin, coll. « 128 ».

MarTin David, 1990, Tongues of Fire. The Explosion of Protestantism in Latin America, Oxford, Blackwell Publishing.

Mearsheimer John J., Walt Stephen M., 2007, Le lobby pro-israélien et la politique étrangère américaine, Paris, La Découverte. [The Israel lobby and US Foreign policy, 2007]

Noll M.A., 2004, "L’influence américaine sur le christianisme évangélique mondial au $\mathrm{XX}^{\mathrm{c}}$ siècle ", in Fath S., (éd.), Le christianisme évangélique : un christianisme de conversion, Turnhout, Brepols, pp. 59-80.

Oтіs George Jr., 1991, The Last of the Giants. Lifting the Veil on Islam and the End Time, Grand Rapids, Chosen Books.

PeretTI Frank E., 1986, This Present Darkness, Wheaton, Crossway Books.

PONCET Patrick, 2007, "MetroMapping ", EspacesTemps.net, Mensuelles, 31.01.2007, http://espacestemps.net/document2162.html. 
84 - ARChives DE SCIENCES SOCIALES DES RELIGIONS

Prudhomme Claude, 2004, Missions chrétiennes et colonisation. $\mathrm{XVI}^{e}$-XX $\mathrm{X}^{e}$ siècle, Paris, Éditions du Cerf.

Roux A., «Les missions protestantes », in Histoire des religions, tome 2, Paris, La PléiadeGallimard, 1972, pp. 1190-1227.

-, 1984, Missions des églises, mission de l'Église. Histoires d'une longue marche, Paris, Éditions du Cerf.

SCAFI A., 1999, " Mapping Eden: Cartographies of the Earthly Paradise ", in Cosgrove D., (ed.), Mappings, Londres, Reaktion Books, pp. 50-70.

Simmel Georg, 1998, La religion, Belval, Circé.

Time Magazine, 30 juin 2003.

TuRner F.J., 1963, La frontière dans l'histoire des États-Unis, Paris, PUF.

WAGNer C. Peter (ed.), 1991, Territorial Spirits: Insights on Strategic-Level Spiritual Warfare from Nineteen Christian Leaders, Ventura, Gospel Light Publications.

-, 1993, Breaking Strongholds in your City. How to use Spiritual Mapping to make your Prayers more Strategic, Effective and Targeted, Ventura, Regal Books.

Woodward David, 1987, " Medieval Mappaemundi ", in Harley J.B., Woodward D., The History of Cartography, vol. 1, Chicago, University of Chicago Press, pp. 286-370.

\section{Sites internet}

http://www.ad2000.org/ - Ad 2000 et Beyond Movement, réseau organisateur du projet " une église pour chaque peuple et l'évangile pour chaque personne pour l'année 2000 » http://ethne.net/ - réseau de données ULPG au service de trois grandes organisations chrétiennes

http://www.joshuaproject.net/ - Joshua Project, base de données sur les ULPG

http://www.isaiah54.org/SpiritualMapping.html - une page sur le spiritual mapping

http://www.mup.org/index.php - Mission to Unreached Peoples, une mission qui œuvre dans la Window $10 / 40$

http://www.uscwm.org/ - US Center for World Mission, stratégie, formation et ressources pour l'œuvre missionnaire ULPG 
LES ÉVANGÉLIQUES CARTOGRAPHIENT LE MONDE - 85

\section{Résumé}

Globalisation et montée en puissance de l'islamisme radical se révèlent de nouveaux enjeux pour les missionnaires évangéliques américains. Dans un souci de viabilité, de crédibilité et d'efficacité, les missions s'arment d'instruments de haute technicité et s'insèrent dans la modernité par la porte de la technologie et du marketing. Le spiritual mapping se présente comme une stratégie pour localiser les besoins en missionnaires, définir les axes d'action, qualifier les cibles. Cette cartographie missionnaire révèle des conceptions propres de l'espace et de l'autre. Replacée dans une perspective historique, on s'aperçoit que, dès les premiers temps, les chrétiens ont développé un intérêt pour la géographie, toujours élaborée à la gloire de Dieu.

Mots-clés : Spiritual mapping, protestantisme, évangéliques, missionnaires, stratégies, globalisation.

\section{Abstract}

Globalization and the rise in power of radical-islamism prove to be new stakes for American evangelic missionaries. For viability, credibility and efficiency's sakes, missions arm themselves with instruments of high-technical nature and filter into modernity through the way of technology and marketing. Spiritual mapping presents itself as a strategy to locate the missionaries' needs, to define axes for action and to describe targets. This missionary mapping reveals peculiar conceptions of space and of the other. Replaced in a historical perspective, we notice that from the beginning, Christians have developed an interest in geography which has always been mapped out to the glory of God.

Key words: Spiritual mapping, protestantism, evangelicals, missionaries, strategies, globalization.

\section{Resumen}

Globalización y aumento del poder del islamismo radical aparecen como los nuevos desafíos para los misioneros evangélicos americanos. En la preocupación por la viabilidad, la credibilidad y la eficacia, las misiones se arman de instrumentos de alta tecnología y se insertan en la modernidad por la puerta de la tecnología y del marketing. El spiritual mapping se presenta como una estrategia para localizar las necesidades en misioneros, definir los ejes de acción, calificar los blancos. Esta cartografía misionera revela concepciones propias del espacio y del otro. Resituada en una perspectiva histórica, percibimos que, desde los primeros tiempos, los cristianos han desarrollado un interés por la geografía, siempre elaborada para gloria de Dios.

Palabras clave: Spiritual mapping, protestantismo, evangélicos, misioneros, estrategias, globalización. 
